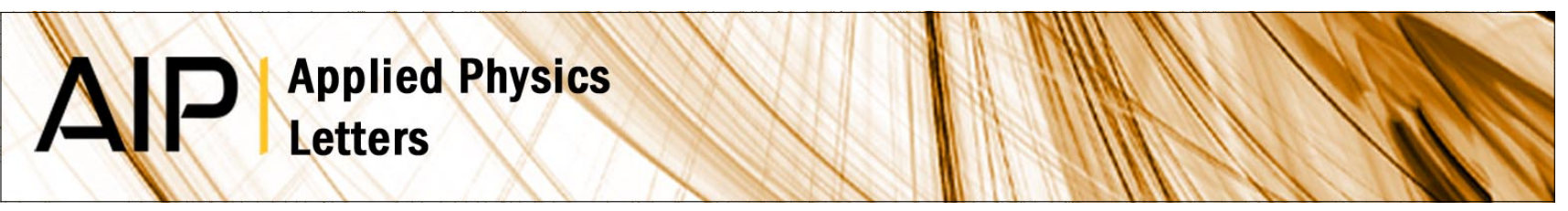

\title{
Reverse mass transport during capping of In0.5Ga0.5As/GaAs quantum
} dots

H. Eisele, Ph. Ebert, N. Liu, A. L. Holmes, and C.-K. Shih

Citation: Appl. Phys. Lett. 101, 233107 (2012); doi: 10.1063/1.4769100

View online: http://dx.doi.org/10.1063/1.4769100

View Table of Contents: http://apl.aip.org/resource/1/APPLAB/v101/i23

Published by the American Institute of Physics.

\section{Additional information on Appl. Phys. Lett.}

Journal Homepage: http://apl.aip.org/

Journal Information: http://apl.aip.org/about/about_the_journal

Top downloads: http://apl.aip.org/features/most_downloaded

Information for Authors: http://apl.aip.org/authors

\section{ADVERTISEMENT}

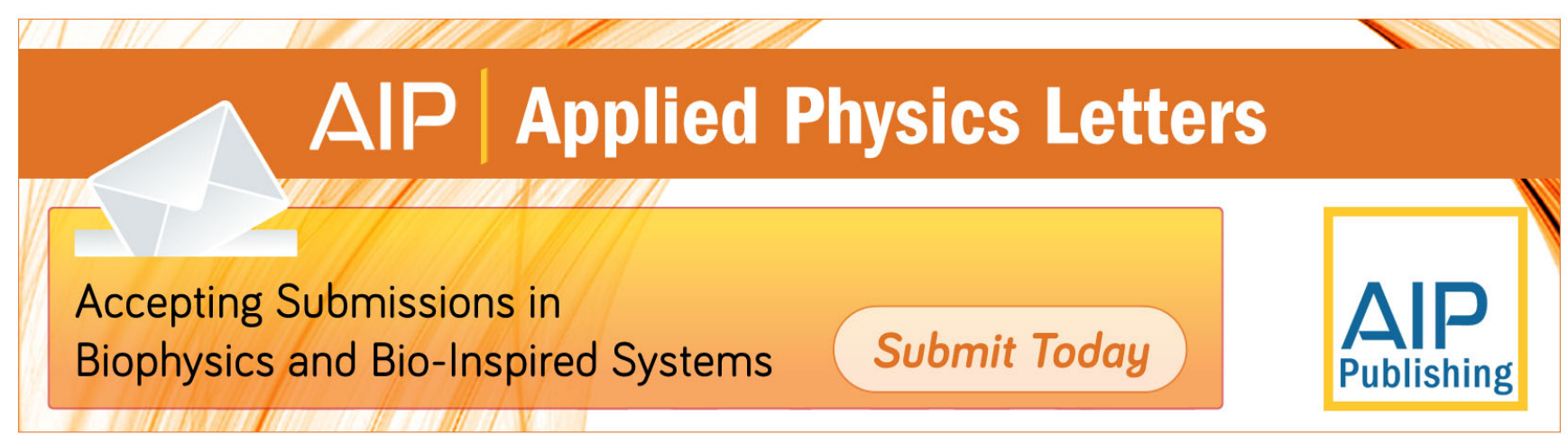




\title{
Reverse mass transport during capping of $\operatorname{In}_{0.5} \mathrm{Ga}_{0.5} \mathrm{As} / \mathrm{GaAs}$ quantum dots
}

\author{
H. Eisele, ${ }^{1,2, a)}$ Ph. Ebert, ${ }^{3}$ N. Liu, ${ }^{1, b)}$ A. L. Holmes, Jr., ${ }^{4, c)}$ and C.-K. Shih ${ }^{1, d)}$ \\ ${ }^{1}$ Department of Physics, The University of Texas at Austin, Austin, Texas 78712, USA \\ ${ }^{2}$ Institut für Festkörperphysik, Technische Universität Berlin, Hardenbergstraße 36, 10623 Berlin, Germany \\ ${ }^{3}$ Peter Grünberg Institut, Forschungszentrum Jülich GmbH, 52425 Jülich, Germany \\ ${ }^{4}$ Department of Electrical Engineering, The University of Texas at Austin, Austin, Texas 78712, USA
}

(Received 17 October 2012; accepted 13 November 2012; published online 4 December 2012)

\begin{abstract}
The rates of indium mass transport between the wetting layer, the quantum dots, and the capping layer are derived from the indium distributions probed by cross-sectional scanning tunneling microscopy of the $\mathrm{In}_{0.5} \mathrm{Ga}_{0.5} \mathrm{As} / \mathrm{GaAs}$ quantum dot system. During capping, a lateral backsegregation from the quantum dots toward the wetting layer is found, reversing the StranskiKrastanov growth mode during quantum dot formation. This lateral back-segregation critically affects the resulting indium distribution in the wetting layer, the apparent segregation coefficients as well as the quantum dot shape. Furthermore, the strain effect on the segregation coefficient is quantified. (C) 2012 American Institute of Physics. [http://dx.doi.org/10.1063/1.4769100]
\end{abstract}

The opto-electronic properties of semiconductor nanostructures, such as quantum dots (QDs), are primarily determined by the spatial arrangement of the individual atoms incorporated within the nanostructure. ${ }^{1-3}$ For example, the distribution of indium atoms in $\mathrm{InAs} / \mathrm{GaAs}$ and $\mathrm{InGaAs} / \mathrm{GaAs}$ QDs influences the effective size and shape of the confinement potential, and hence determines their lasing properties. ${ }^{4-8}$ In order to design optimized semiconductor nanostructures based on a bottom-up approach, it is crucial to unravel and understand the detailed physical processes affecting the incorporation and redistribution of atoms during growth and capping. ${ }^{9,10}$ However, rather little is known about these atomic processes even for the widely investigated InAs/GaAs and InGaAs/GaAs QD systems, because of the difficulty to directly access and probe them.

For InGaAs material deposition on $\operatorname{GaAs}\left(\begin{array}{lll}0 & 0 & 1\end{array}\right)$, an inverted cone composition profile occurs in the QDs during growth. ${ }^{11,12}$ Furthermore, capping by GaAs leads to flat $\left(\begin{array}{lll}0 & 0 & 1\end{array}\right)$ top facets and to a vertical indium segregation destroying the desired atomic sharpness of the top interface, in both, the InGaAs/GaAs and the InAs/GaAs systems. ${ }^{10-17}$ In the pure InAs/GaAs QD system, the presence of an additional lateral segregation was suggested. ${ }^{10}$ However, widely deviating segregation coefficients were observed for the QD and wetting layer (WL) material within the capping layer (CL). ${ }^{18-21}$ Hence, it is completely unclear how the vertical and lateral indium mass transport during growth and capping are really driven and how they are related to each other. Furthermore, at present, it is unclear where the material from the removed apex goes to, i.e., the QD flanks or the WL.

In this letter, we identify the physical processes of indium mass transport being active during growth and capping of the InGaAs/GaAs QD model system by cross-sectional scanning tunneling microscopy (XSTM). Based on a mapping of the distribution of indium atoms with atomic resolution, we

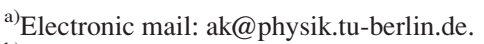

${ }^{b)}$ Present address: Freescale Semiconductor, Inc., Austin, Texas 78721, USA.

${ }^{c)}$ Present address: Electrical and Computer Engineering Department, University of Virgina, 395 McCormick Rd, Charlottesville, Virginia 22904, USA.

d) Electronic mail: shih@ physics.utexas.edu.
}

derived the rates of mass transport between the WL, the QDs, and the CL. We found that the lateral back-segregation from the QDs toward the WL during capping is a crucial component to govern the resulting concentration in the $\mathrm{WL}$ and $\mathrm{CL}$ as well as the QD shape. Hence, during capping a reversed mass transport with respect to the Stranski-Krastanov growth mode ${ }^{22}$ occurs.

For the investigation of the mass transport, we investigated $\mathrm{In}_{0.5} \mathrm{Ga}_{0.5}$ As QD layers with a nominal deposition of 5,6 , and $18 \mathrm{ML}$ on $\mathrm{GaAs}\left(\begin{array}{lll}0 & 0 & 1\end{array}\right)$ at $510^{\circ} \mathrm{C}$ using migration enhanced epitaxy. ${ }^{11}$ During growth, In and Ga are supplied alternating in discrete amounts of $0.5 \mathrm{ML}$, each followed by $7 \mathrm{~s}$ of As flux. For the GaAs capping layer, the Ga and As fluxes were supplied continuously. The atomic mapping by XSTM was performed on $\left(\begin{array}{lll}1 & 1 & 0\end{array}\right)$ planes obtained in situ in ultra-high vacuum $\left(p<10^{-8} \mathrm{~Pa}\right)$. In principle, the indium concentration can be derived from measuring the local lateral lattice parameter along growth direction ${ }^{23}$ or-if the concentration is low enough - by counting the single bright spots in every layer from the images. ${ }^{24,25}$ Here, we used the latter method on multiple images in order to get sufficient significance of our data.

Figure 1(a) shows an overview empty-state XSTM image of a QD obtained after deposition of $18 \mathrm{ML}$ of $\mathrm{In}_{0.5} \mathrm{Ga}_{0.5} \mathrm{As}$. The QD is confined by a truncated pyramidal shape (solid lines). Within the QD, the inverted cone shape of the indium distribution is visible (dashed lines). Figure 1(b) shows the high resolution empty-state XSTM image of the central area of the QD. At the image, the atomic rows along $\left[\begin{array}{lll}1 & 1 & 0\end{array}\right]$ representing every second deposited monolayer [(lll $\left.\begin{array}{ll}0 & 1\end{array}\right)$ planes $]$ are visible. In addition, atomic sized bright spots indicate the presence of individual indium atoms. The spatial position of every indium atom within the topmost layer of the cleavage surface can be clearly identified. ${ }^{24}$ Figure 1 (c) shows the indium concentration at the QD center along growth direction, obtained from the red dashed-dotted area in Fig. 1(b). The indium concentration within the QD increases strongly along growth direction, dropping sharply at its top. In addition, an indium segregation tail above the QD is visible. This shape of the indium concentration profile agrees with the theoretical model given in Ref. 11. 


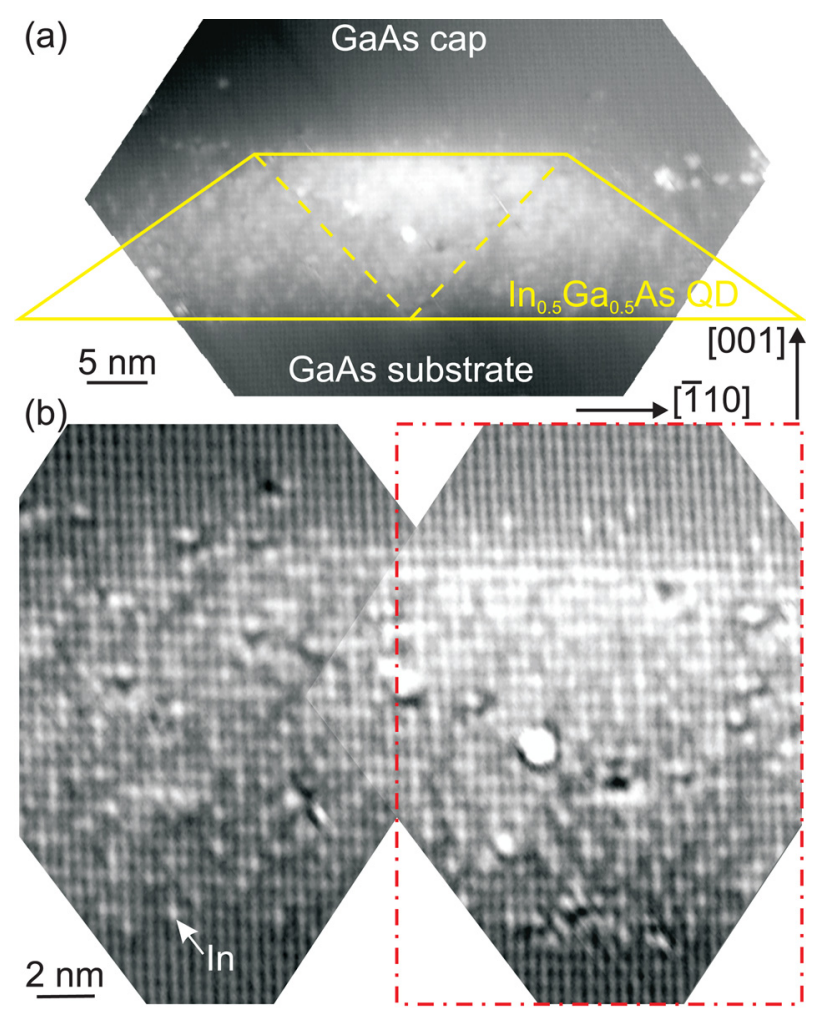

(c)

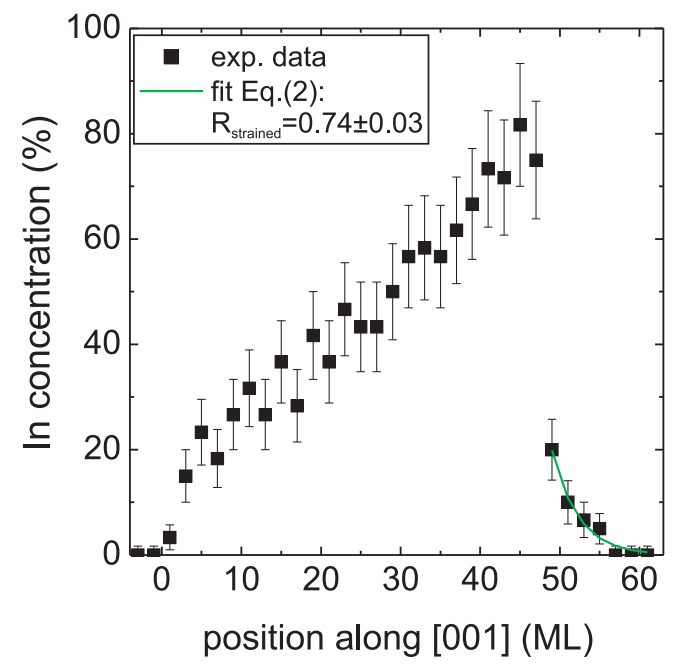

FIG. 1. (a) Cross-sectional scanning tunneling microscope image of an $\mathrm{In}_{0.5} \mathrm{Ga}_{0.5} \mathrm{As} / \mathrm{GaAs} \mathrm{QD}$. The overall shape of the QD is indicated by (yellow) solid lines. The internal inverted cone indium distribution is marked by (yellow) dashed lines. (b) Composite high resolution XSTM image of the central area of the QD with enhanced contrast to illustrate the positions of every individual indium atom (bright atomic contrast). (c) Indium concentration in the QD center along the growth direction extracted from the (red) dashed-dotted area in (b). The indium concentration increases along the growth direction and a segregation tail is visible above the QD top. The (green) solid line is a fit of the segregation tail.

Figures 2(a1), 2(b1), and 2(c1) show XSTM images of the wetting layers far away from QD for 5, 6, and $18 \mathrm{ML}$ $\mathrm{In}_{0.5} \mathrm{Ga}_{0.5} \mathrm{As}$ deposition, respectively. From these and additional images, the indium concentration profiles along the growth direction were determined (Figs. 2(a2), 2(b2), and 2(c2), respectively). Each indium concentration profile exhibits a strong increase right after the start of $\operatorname{In}_{0.5} \mathrm{Ga}_{0.5} \mathrm{As}$ deposition. After a certain deposition amount (including the following capping), the indium concentration decreases and segregation tails are visible. ${ }^{26,27}$ However, quantitatively the indium concentration profiles differ significantly with increasing $\operatorname{In}_{0.5} \mathrm{Ga}_{0.5}$ As deposition amounts.

In order to unravel the physical process leading to these concentration profiles, the mass transport rate equations are derived. Starting from the model given in Ref. 27 for quantum wells (without quantum dots), the indium concentration $c_{\text {In }}$ can be described by

$$
c_{\text {In }}(n)=c_{0} \cdot\left(1-R^{n}\right)
$$

for the $n$th monolayer along the growth direction with $0<n<N$, where $N$ is the number of deposited $\operatorname{In}_{0.5} \mathrm{Ga}_{0.5} \mathrm{As}$ monolayers. For $n>N$ (capping), where no further indium is deposited, a segregation tail develops, where the indium concentration decreases according to

$$
c_{\mathrm{In}}(n)=c_{0} \cdot\left(1-R^{N}\right) \cdot R^{(n-N)} .
$$

$R$ is the segregation coefficient and $c_{0}$ is the In concentration in the deposited material, i.e., 0.5 for $\operatorname{In}_{0.5} \mathrm{Ga}_{0.5} \mathrm{As}$.

The calculated indium concentration profiles expected for a quantum well structure without quantum dots [Eqs. (1) and (2)] are plotted for the nominal $\operatorname{In}_{0.5} \mathrm{Ga}_{0.5}$ As deposition of 5, 6, and $18 \mathrm{ML}$ as blue dashed lines in Figs. 2(a2), 2(b2) and 2(c2), respectively, using $R=0.78$. The measured indium concentration (black squares) follows (within the error bars) only the calculated segregation profile curve in the case of $5 \mathrm{ML}$ of $\operatorname{~n}_{0.5} \mathrm{Ga}_{0.5}$ As deposition. Indeed, a fit of the segregation tail in the case of $5 \mathrm{ML}$ of $\mathrm{In}_{0.5} \mathrm{Ga}_{0.5} \mathrm{As}$ deposition using Eq. (2) [green dashed-dotted line in Fig. 2(a2)] yields a segregation coefficient of $R_{5 \mathrm{ML}}=0.78 \pm 0.05$ and $\mathrm{a}$ deposited indium concentration of $c_{0}=0.56 \pm 0.07$. This indicates that no indium is accumulated in quantum dots, in agreement with the experimentally observed absence of quantum dots in this layer.

In contrast, for $6 \mathrm{ML} \operatorname{In}_{0.5} \mathrm{Ga}_{0.5}$ As deposition, the fit of the segregation tail [green dashed-dotted line in Fig. 2(b2)] yields the same segregation coefficient of $R_{6 \mathrm{ML}}=0.78$ \pm 0.03 , but a smaller deposited indium concentration of only $c_{0}=0.43 \pm 0.03$ as compared with the nominal 0.5. This reduced indium concentration indicates a mass transfer from the wetting layer into the quantum dots, resulting in a lower indium content at the positions 3,5 , and $7 \mathrm{ML}$, where the capping starts. Note that the nominal total deposition of $6 \mathrm{ML}$ is close to the critical thickness of $6.4 \pm 0.8 \mathrm{ML}$, at which quantum dot formation starts for $\operatorname{In}_{0.5} \mathrm{Ga}_{0.5} \mathrm{As}$ $/ \mathrm{GaAs},{ }^{28}$ corroborating the above conclusions.

For the $18 \mathrm{ML} \operatorname{In}_{0.5} \mathrm{Ga}_{0.5}$ As deposition, the blue dashed curve in Fig. 2(c2) shows the expected indium profile in the wetting layer based on a segregation coefficient $R=0.78$. It deviates even more than for the $6 \mathrm{ML}$ case from the experimental data. Instead, a linear decrease of the In concentration within the wetting layer (grey dashed line) is observed, again demonstrating the now more pronounced mass transport due to quantum dot evolution. It is followed by an exponential decay in the cap layer above the position of $19 \mathrm{ML}$, which is again the segregation tail. A fit of the segregation tail using Eq. (2) [green dashed-dotted line in Fig. 2(c2)], yields a deposited indium concentration in the wetting layer of only $c_{0}=0.10 \pm 0.01$, i.e., much lower than the nominal one of 0.5 . 
$5 \mathrm{ML}$
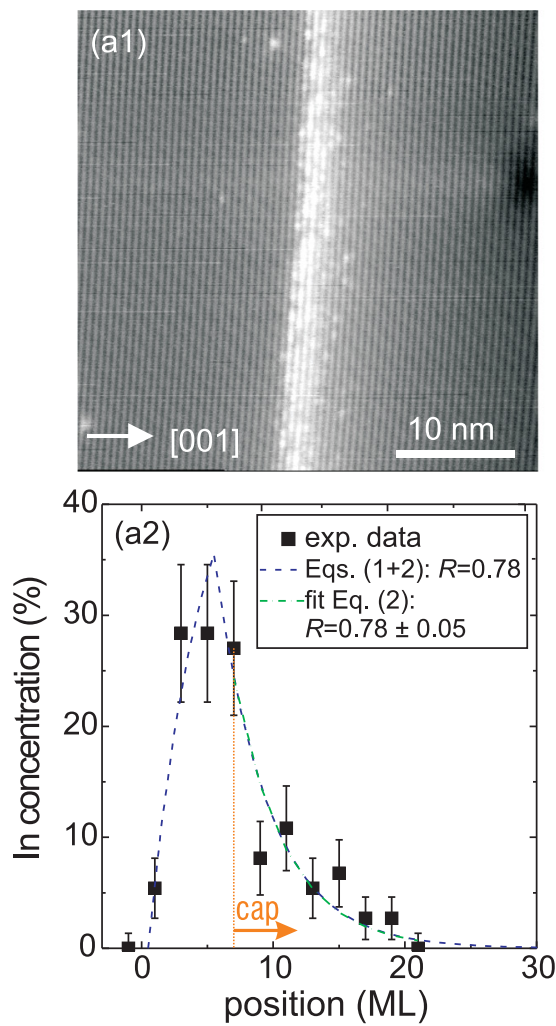

$6 \mathrm{ML}$
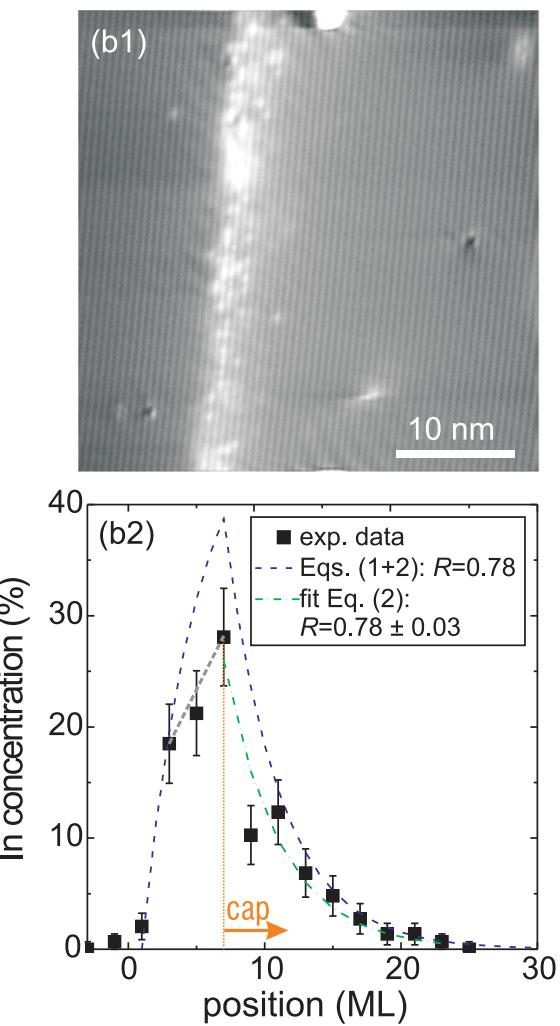

$18 \mathrm{ML}$
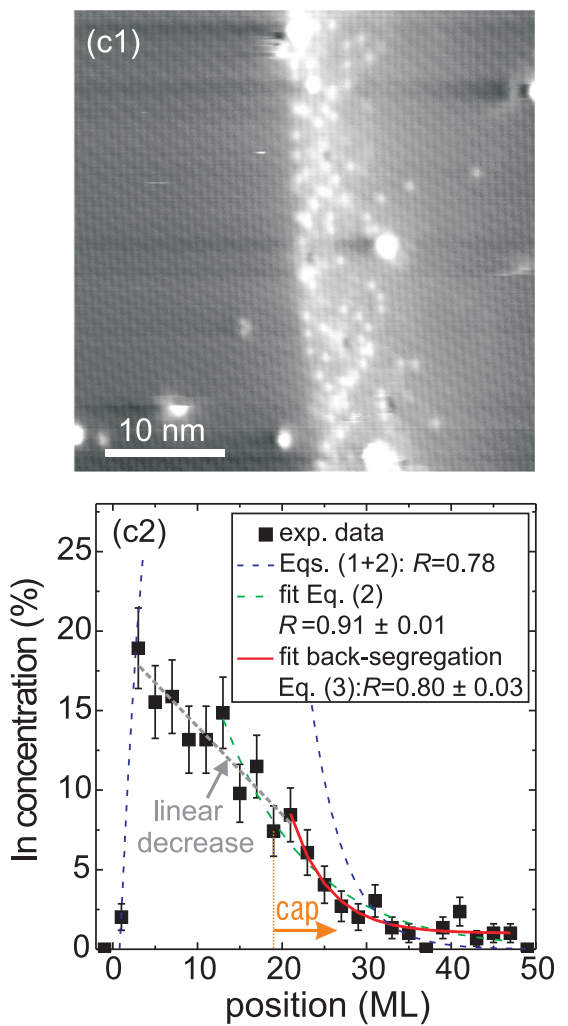

FIG. 2. Cross-sectional scanning tunneling microscopy images of the wetting layers after deposition of (a1) 5 , (b1) 6 , and (c1) $18 \mathrm{ML}$ of In $0.5 \mathrm{Ga} 0.5 \mathrm{As}$ on GaAs. The corresponding indium concentration profiles are shown below each XSTM image in frames (a2), (b2), and (c2), respectively. The different lines are fits to the experimental data using different models.

Furthermore, the segregation coefficient of $R_{18 \mathrm{ML}}=$ $0.91 \pm 0.01$ obtained from the fit using Eq. (2) [green dasheddotted line in Fig. 2(c2)] deviates significantly from the above values and from typical values known from literature for the used growth conditions (for the $\mathrm{In}(\mathrm{Ga}) \mathrm{As} / \mathrm{GaAs}$ system $R \approx 0.75-0.80){ }^{18,20,21}$ But, there is no physical reason for a change of the segregation coefficient. Thus, the model of simple vertical indium segregation from one to the following layer is not describing the resulting indium concentration profile accurately enough. This indicates the neglect of relevant parts of the indium mass transport during capping process.

In order to identify the additional mass transport mechanism, it should be remarked that before capping the pyramid shaped quantum dots elastically relax approximately half of their strain energy. ${ }^{29}$ Capping of the quantum dots by the GaAs matrix material reduces the ability of relaxation. Hence, the strain energy within the quantum dotespecially at its apex-increases significantly until the pyramidal QD apex is removed. This results in the formation of a flat $(001)$ top facet, ${ }^{10}$ also visible in Fig. 1(a), in order to limit the strain energy. The apex removal process initiates a lateral indium mass transport away from the quantum dot toward the wetting layer regions (Fig. 3). This process requires the introduction of a lateral mass transport in a quantitative description of the indium concentration profiles in capping layers. The mass transport can be considered as a lateral back-segregation from the quantum dots toward the wetting layer region, yielding an indium concentration profile of

$$
c_{\mathrm{In}}(n)=c_{0} \cdot\left(1-R^{N}\right) \cdot R^{(n-N)} \cdot(1-R)+c_{\mathrm{bs}} \cdot\left(1-R^{(n-N)}\right)
$$

for $n>N$. $c_{\mathrm{bs}}$ represents the indium concentration added to each capping monolayer because of lateral back-segregation from the QD. Fitting the experimental data in Fig. 2(c2) using Eq. (3) (red solid curve) yields $R=0.80 \pm 0.03$, $c_{0}=0.65 \pm 0.14$, and $c_{\mathrm{bs}}=0.010 \pm 0.003$. The vertical segregation coefficient is now also in agreement with the above values of $R=0.78$ for the 5 and $6 \mathrm{ML}$ depositions. The lateral back-segregation rate from the QD towards the WL is $c_{\text {bs }}=0.01$ per deposited ML of GaAs cap material, until the QD is completely covered [approximately at a position of about 50 ML in Figs. 1(c) and 2(c2)]. This illustrates that the inclusion of the back-segregation in the data analysis is crucial for obtaining accurate vertical segregation coefficients. Otherwise, excessively large apparent segregation coefficients ${ }^{19}$ are obtained. Furthermore, it shows that the indium

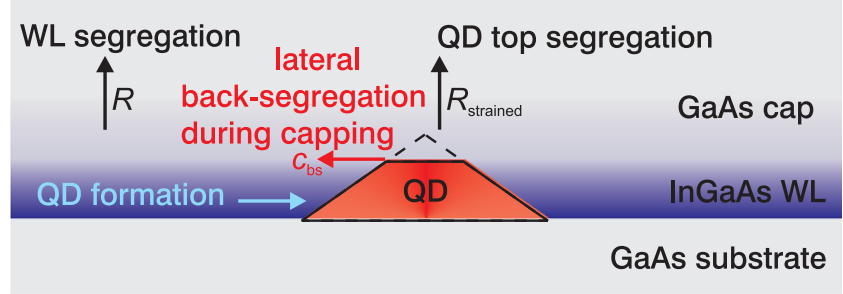

FIG. 3. Schematic illustration of the different atomic processes involved in the quantum dot formation during growth and capping. 
removed from the apex is redistributed into the capping layer above the wetting layer.

At this stage, we discuss the total amount of indium within the WL. Integrating the indium concentration curves in Fig. 2 up to the position, at which the capping starts, leads to $1.4 \mathrm{ML}$ of pure InAs for both, the $5 \mathrm{ML}$ and $6 \mathrm{ML}$ cases. This is consistent with critical thickness for QD evolution for pure InAs deposition. ${ }^{28}$ In the $18 \mathrm{ML}$ case, we find $2.4 \pm 0.3 \mathrm{ML}$ of pure InAs material within the WL. This indicates that the gradient of strain energy is reduced for low indium concentrations $(\leq 15 \%)$ present in the WL of the $18 \mathrm{ML}$ case, leading to the characteristic linear decrease of the indium concentration within the WL [Fig. 2(c2)]. Hence, the indium mass transport from the WL to the QD is also reduced.

Finally, the indium segregation within the CL above the QDs allows to probe the effect of a lateral strained lattice on the segregation coefficient. The local lattice parameter above the QD is enlarged as compared with above the WL. From the analysis of the segregation tail in Fig. 1(c), a segregation coefficient of $R_{\text {strained }}=0.74 \pm 0.03$ is determined. This reduced segregation coefficient reflects the reduced straininduced driving force for indium segregation in laterally expanded GaAs lattices.

With these results, it becomes clear that all mass transport processes during growth and capping are strain driven: In order to lower the total energy during growth, first QDs evolve, at which the strain can relax locally. During capping, the additionally introduced strain energy drives the strained material back toward the WL region, but not into the QD flanks as in the case of pure InAs depositions. ${ }^{10}$ Independently from these two processes, the strained material furthermore segregates along growth direction, which is also a strain driven process since the segregation coefficient above the QDs is smaller as compared with above the WL.

In conclusion, we probed the indium distribution in quantum dots and in the wetting layer of the $\operatorname{In}_{0.5} \mathrm{Ga}_{0.5} \mathrm{As} / \mathrm{GaAs}$ system using cross-sectional scanning tunneling microscopy. From the analysis of the spatial distribution of individual indium atoms, we derived the rates of mass transport between the WL, the QDs, and the CL. We found that the lateral backsegregation of $0.010 \pm 0.003 \mathrm{ML}$ indium per ML of capping material from the QDs to the WL region during capping is a crucial component to understand the resulting indium distribution in the WL as well as the QD shape. In addition, the vertical segregation constants on unstrained and strained lattices are quantified and we demonstrated their correct determination.
The authors thank J. Tersoff for fruitful discussions as well as S. Govindaraju and O. Baklenov for sample growth. This work was supported by NFS-DMR 9402938 and NFS Science and Technology Center Grant No. CHE 8920120, as well as by the Alexander von Humboldt-Foundation.

${ }^{1}$ Semiconductor Quantum Dots, edited by Y. Masumoto and T. Takagahara (Springer, Berlin, 2002).

${ }^{2}$ D. Bimberg, J. Phys. D 38, 2055 (2005)

${ }^{3}$ Semiconductor Nanostructures, edited by D. Bimberg (Springer, Berlin, 2008).

${ }^{4}$ L.-W. Wang, J. Kim, and A. Zunger, Phys. Rev. B 59, 5678 (1999).

${ }^{5}$ O. Stier, M. Grundmann, and D. Bimberg, Phys. Rev. B 59, 5688 (1999).

${ }^{6}$ O. Stier, R. Heitz, A. Schliwa, and D. Bimberg, Phys. Status Solidi A 190, 477 (2002).

${ }^{7}$ G. Bester, X. Wu, D. Vanderbilt, and A. Zunger, Phys. Rev. Lett. 96, 187602 (2006).

${ }^{8}$ A. Schliwa, M. Winkelnkemper, and D. Bimberg, Phys. Rev. B 79, 075443 (2009).

${ }^{9}$ S. Tsukamoto, T. Honma, G. Bell, A. Ishii, and Y. Arakawa, Small 2, 386 (2006).

${ }^{10}$ H. Eisele, A. Lenz, R. Heitz, R. Timm, M. Dähne, Y. Temko, T. Suzuki, and K. Jacobi, J. Appl. Phys. 104, 124301 (2008).

${ }^{11}$ N. Liu, J. Tersoff, O. Baklenov, A. Holmes, and C.-K. Shih, Phys. Rev. Lett. 84, 334 (2000).

${ }^{12}$ A. Lenz, R. Timm, H. Eisele, Ch. Hennig, S. K. Becker, R. L. Sellin, U. W. Pohl, D. Bimberg, and M. Dähne, Appl. Phys. Lett. 81, 5150 (2002).

${ }^{13}$ B. Legrand, B. Grandidier, J. Nys, D. Stiévenard, J. Gérard, and V. Thierry-Mieg, Appl. Phys. Lett. 73, 96 (1998).

${ }^{14}$ H. Eisele, O. Flebbe, T. Kalka, C. Preinesberger, F. Heinrichsdorff, A. Krost, D. Bimberg, and M. Dähne-Prietsch, Appl. Phys. Lett. 75, 106 (1999).

${ }^{15}$ B. Lita, R. Goldman, J. Phillips, and P. Bhattacharya, Appl. Phys. Lett. 74, 2824 (1999).

${ }^{16}$ J. Márquez, L. Geelhaar, and K. Jacobi, Appl. Phys. Lett. 78, 2309 (2001).

${ }^{17}$ H. Eisele and K. Jacobi, Appl. Phys. Lett. 90, 129902 (2007).

${ }^{18}$ A. Rosenauer, D. Gerthsen, D. Van Dyck, M. Arzberger, G. Böhm, and G. Abstreiter, Phys. Rev. B 64, 245334 (2001).

${ }^{19}$ P. Offermans, P. Koenraad, R. Nötzel, J. Wolter, and K. Pierz, Appl. Phys. Lett. 87, 111903 (2005).

${ }^{20}$ D. Litvinov, D. Gerthsen, A. Rosenauer, M. Schowalter, T. Passow, P. Feinäugle, and M. Hetterich, Phys. Rev. B 74, 165306 (2006).

${ }^{21}$ A. Lenz, H. Eisele, J. Becker, L. Ivanova, E. Lenz, F. Luckert, K. Pötschke, A. Strittmatter, U. W. Pohl, D. Bimberg, and M. Dähne, Appl. Phys. Express 3, 105602 (2010).

${ }^{22}$ I. N. Stranski and L. Krastanow, Sitzungsber. Akad. Wiss. Wien, Math.-Naturwiss. Kl., Abt. IIB 146, 797 (1938).

${ }^{23}$ H. Eisele, R. Timm, A. Lenz, Ch. Hennig, M. Ternes, S. K. Becker, and M. Dähne, Phys. Status Solidi C 0, 1129 (2003).

${ }^{24}$ K.-J. Chao, C.-K. Shih, D. Gotthold, and B. Streetman, Phys. Rev. Lett. 79, 4822 (1997).

${ }^{25}$ H. Eisele, O. Flebbe, T. Kalka, F. Heinrichsdorff, A. Krost, D. Bimberg, and M. Dähne-Prietsch, Phys. Status Solidi B 215, 865 (1999).

${ }^{26}$ W. Ni, J. Knall, M. Hasan, G. Hansson, J. Sundgren, S. Barnett, L. Markert, and J. Greene, Phys. Rev. B 40, 10449 (1989).

${ }^{27}$ K. Muraki, S. Fukatsu, Y. Shiraki, and R. Ito, Appl. Phys. Lett. 61, 557 (1992).

${ }^{28}$ H. Eisele and M. Dähne, J. Cryst. Growth 338, 103 (2012).

${ }^{29}$ O. Stier, personal communication (2001). 